\title{
INVERSE LIMITS, ENTROPY AND WEAK ISOMORPHISM FOR DISCRETE DYNAMICAL SYSTEMS
}

\author{
BY
}

\author{
JAMES R. BROWN
}

\begin{abstract}
A categorical approach is taken to the study of a single measurepreserving transformation of a finite measure space and to inverse systems and inverse limits of such transformations. The questions of existence and uniqueness of inverse limits are settled. Sină’s theorem on generators is recast and slightly extended to say that entropy respects inverse limits, and various known results about entropy are obtained as immediate corollaries, e.g. systems with quasi-discrete or quasi-periodic spectrum have zero entropy. The inverse limit $\Phi$ of an inverse system $\left\{\Phi_{\alpha}: \alpha \in J\right\}$ of dynamical systems is (1) ergodic, (2) weakly mixing, (3) mixing (of any order) iff each $\Phi_{\alpha}$ has the same property. Finally, inverse limits are used to lift a weak isomorphism of dynamical systems $\Phi_{1}$ and $\Phi_{2}$ to an isomorphism of systems $\Phi_{1}$ and $\Phi_{2}$ with the same entropy.
\end{abstract}

1. Introduction. By a discrete, abstract dynamical system (or simply dynamical system) we shall mean a quadruple $\Phi=(X, \mathscr{B}, \mu, \varphi)$, where $X$ is a nonempty set, $\mathscr{B}$ is a $\sigma$-algebra of subsets of $X, \mu$ is a normalized (total measure one) measure defined on $\mathscr{B}$, and $\varphi$ is a measurable and measure-preserving (but not necessarily invertible) mapping of $X$ into itself.

For practical reasons we shall, in fact, be interested not in such quadruples but in equivalence classes of such quadruples. Clearly, there is no reason to distinguish between the mapping $x \rightarrow 2 x$ mod 1 of the unit interval and the mapping $z \rightarrow z^{2}$ of the unit circle in the complex plane. Moreover, we shall want to identify, for example, the measure space $X=\{0,1\}$, where $\mathscr{B}$ is the class of all subsets of $X$ and $\mu$ is the counting function, with the measure space $X=[0,1]$,

$$
\mathscr{B}=\left\{\varnothing,\left[0, \frac{1}{2}\right],\left[\frac{1}{2}, 1\right], X\right\},
$$

where $\mu$ is the restriction to $\mathscr{B}$ of Lebesgue measure. For these reasons we shall (more or less consistently) identify any two dynamical systems $\Phi=(X, \mathscr{B}, \mu, \varphi)$ and $\Phi^{\prime}=\left(X^{\prime}, \mathscr{B}^{\prime}, \mu^{\prime}, \varphi^{\prime}\right)$ for which there exists a mapping $\psi^{*}: \mathscr{B}^{\prime} \rightarrow \mathscr{B}$ which is one-to-one and onto, and which satisfies

and

$$
\mu\left(\psi^{*} B^{\prime}\right)=\mu^{\prime}\left(B^{\prime}\right) \quad\left(B^{\prime} \in \mathscr{B}^{\prime}\right)
$$

$$
\mu\left(\varphi^{-1}\left(\psi^{*} B^{\prime}\right) \Delta \psi^{*}\left(\varphi^{\prime-1} B^{\prime}\right)\right)=0 \quad\left(B^{\prime} \in \mathscr{B}^{\prime}\right) .
$$

Received by editors January 8, 1970.

AMS 1970 subject classifications. Primary 28A65; Secondary 18A30, 18B99, $20 \mathrm{~K} 30$.

Key words and phrases. Inverse limits, dynamical systems, measure-preserving transformation, factor, invariant subalgebra, weakly isomorphic, direct product, bounded inverse system, Lebesgue system, discrete spectrum, exact system, natural extension, disjoint, ergodic, weakly mixing, mixing (of any order), quasi-discrete spectrum, quasi-periodic spectrum. 
Of course, if $\psi: X \rightarrow X^{\prime}$ is a measure-preserving, invertible map of $X$ essentially onto $X^{\prime}$, such that $\psi^{-1}$ is also measurable and $\psi \varphi=\varphi^{\prime} \psi(\bmod z e r o)$, then $\psi^{*}(B)$ $=\psi^{-1}(B)$ effects such an identification.

The principal advantage of this identification is that we may now assume, by an appropriate replacement of the underlying space or spaces, that set mappings $\rho^{*}: \mathscr{B}^{\prime} \rightarrow \mathscr{B}$ connecting two dynamical systems $\Phi$ and $\Phi^{\prime}$ are in fact given by point mappings $\rho: X \rightarrow X^{\prime}$ (see Halmos and von Neumann [8] and D. Maharam [9]).

We shall say that the dynamical system $\Phi_{1}=\left(X_{1}, \mathscr{B}_{1}, \mu_{1}, \varphi_{1}\right)$ is a factor of the system $\Phi=(X, \mathscr{B}, \mu, \varphi)$ if there exists a measure-preserving map $\psi: X \rightarrow X_{1}$ satisfying $\psi \varphi=\varphi_{1} \psi(\bmod z e r o)\left({ }^{1}\right)$. In this case we write $\Phi_{1} \mid \Phi$ and $\psi: \Phi \rightarrow \Phi_{1}$ or $\Phi \stackrel{\psi}{\rightarrow} \Phi_{1}$. Note that by our identification we may assume that $X=X_{1}, \mathscr{B}_{1} \subseteq \mathscr{B}$ and $\mu_{1}$ and $\varphi_{1}$ are merely the restrictions of $\mu$ and $\varphi$. Of course, $\mathscr{B}_{1}$ is then an invariant sub- $\sigma$-algebra of $\mathscr{B}$ in the sense that $\varphi^{-1} \mathscr{B}_{1} \subseteq \mathscr{B}_{1}$ (mod zero). The study of factors of $\Phi$ is thus reduced to the study of invariant sub- $\sigma$-algebras of $\mathscr{B}$. In this regard, note that the factor $\Phi_{1}$ is an invertible system in the sense that $\varphi_{1}$ has an equivalent representation as an invertible, bimeasurable measure-preserving transformation iff $\mathscr{B}_{1}$ is totally invariant, that is $\varphi^{-1} \mathscr{B}_{1}=\mathscr{B}_{1}(\bmod$ zero).

It might be imagined that because of our identification of equivalent systems, if $\Phi_{1}$ is a factor of $\Phi_{2}$ and $\Phi_{2}$ is a factor of $\Phi_{1}$, then $\Phi_{1}=\Phi_{2}$. However, it is not known whether this is true (see [14]). Under these conditions we follow Sinaĭ and say that $\Phi_{1}$ and $\Phi_{2}$ are weakly isomorphic.

A closely related concept to that of factor is direct product. If $\Phi_{1}=\left(X_{1}, \mathscr{B}_{1}, \mu_{1}, \varphi_{1}\right)$ and $\Phi_{2}=\left(X_{2}, \mathscr{B}_{2}, \mu_{2}, \varphi_{2}\right)$ are dynamical systems, we define their direct product $\Phi=\Phi_{1} \otimes \Phi_{2}$ by $\Phi=\left(X_{1} \times X_{2}, \mathscr{B}_{1} \times \mathscr{B}_{2}, \mu_{1} \times \mu_{2}, \varphi_{1} \times \varphi_{2}\right)$, where $\left(\varphi_{1} \times \varphi_{2}\right)\left(x_{1}, x_{2}\right)$ $=\left(\varphi_{1} x_{1}, \varphi_{2} x_{2}\right)$. More generally, if $\Phi_{\alpha}=\left(X_{\alpha}, \mathscr{B}_{\alpha}, \mu_{\alpha}, \varphi_{\alpha}\right)$ is a dynamical system for each $\alpha \in J$, we define the direct product $\Phi=\bigotimes_{\alpha \in J} \Phi_{\alpha}$ by taking the product measure structure and defining

$$
\varphi(x)=y \quad \text { where } y_{\alpha}=\varphi_{\alpha} x_{\alpha} .
$$

We shall make use also of customary symbols such as $\Phi_{1} \otimes \Phi_{2} \otimes \cdots \otimes \Phi_{n}$ and $\bigotimes_{n=1}^{\infty} \Phi_{n}$.

If $\Phi=\Phi_{1} \otimes \Phi_{2}$, then clearly $\Phi_{1}$ is a factor of $\Phi$. We shall say that it is a direct factor.

For a further discussion of the "arithmetic" of dynamical systems see [5]. We shall be more interested in some aspects of the "calculus" of such systems, as exemplified by the next section.

A word or two about originality of the results in this paper seems to be in order. We have attempted to make our discussion of inverse systems and their entropy more or less self-contained. Our approach is categorical and from that point of

(') We use the phrase "mod zero" in the established sense that the relation holds after discarding appropriate sets of measure zero. The presence of this modifier will be assumed throughout when not specifically denied or inappropriate. 
view the definitions and arguments are quite standard, though perhaps new to ergodic theory. Theorem 1 is new but not terribly exciting. It seems necessary for completeness. Theorem 2 is a new way of stating a theorem of J. R. Choksi [4] with some extra maps floating around. Theorem 3 is new. It elaborates on the representation theorem of Halmos and von Neumann [8] and anticipates the results of the present author on systems with quasi-discrete spectrum [3]. Theorems 4 through 7 are technical necessities.

Theorem 8 is an obvious reformulation of Sinaís important theorem on generators. It is the most natural form of that theorem and contains as well the various extensions given by Billingsley [2]. This is, of course, a continuity theorem, and it is worth noting that countability of the inverse system is not required.

Theorem 9 is new.

2. Inverse limits. The direct product of infinitely many dynamical systems may be thought of as a limit of finite products in a way which will become clear in the following. On the other hand, the slightly more general notion of inverse limit is useful in calculation of entropy (see §3) and the analysis of complex dynamical systems (see the examples below and the paper [3]). We shall give a categorical definition (i.e. one involving only maps between dynamical systems) thus avoiding temporarily some of the sticky problems of existence. Note that the direct product in the previous section could also have been defined categorically.

By an inverse system of dynamical systems we shall mean a triple $\left(J, \Phi_{\alpha}, \psi_{\alpha \alpha^{\prime}}\right)$ such that $J$ is a directed set, for each $\alpha \in J, \Phi_{\alpha}$ is a dynamical system, and for each pair $\alpha, \alpha^{\prime} \in J$ with $\alpha<\alpha^{\prime}$, we have $\psi_{\alpha \alpha^{\prime}}: \Phi_{\alpha^{\prime}} \rightarrow \Phi_{\alpha}$. That is $\Phi_{\alpha} \mid \Phi_{\alpha^{\prime}}$ whenever $\alpha<\alpha^{\prime}$. An upper bound for such a system is a dynamical system $\Phi$ such that $\Phi_{\alpha} \mid \Phi$ for each $\alpha \in J$, say $\rho_{\alpha}: \Phi \rightarrow \Phi_{\alpha}$, and such that, moreover, for each $\alpha, \alpha^{\prime} \in J$ with $\alpha<\alpha^{\prime}$ the diagram

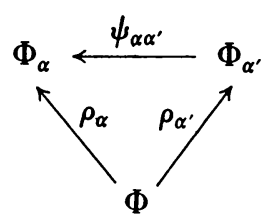

commutes. Finally, an inverse limit $\Phi$ of the inverse system $\left(J, \Phi_{\alpha}, \psi_{\alpha \alpha^{\prime}}\right)$ is an upper bound with maps $\hat{\rho}_{\alpha}: \dot{\Phi} \rightarrow \Phi_{\alpha}$ which is a factor of every other upper bound. That is whenever $\Phi$ is an upper bound with maps $\rho_{\alpha}: \Phi \rightarrow \Phi_{\alpha}$, there exists a map $\sigma: \Phi \rightarrow \Phi$ such that the diagram

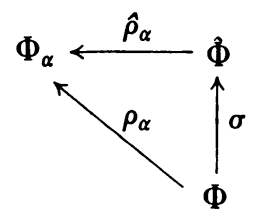


commutes for each $\alpha \in J$. In this case we write

$$
\hat{\Phi}=\underset{\alpha \in J}{\operatorname{inv}} \lim \Phi_{\alpha} \quad \text { or } \quad \hat{\Phi}=\text { inv } \lim \left(\Phi_{\alpha \in J}, \psi_{\alpha \alpha^{\prime}}\right) .
$$

Clearly, if $\Phi=(X, \mathscr{B}, \mu, \varphi)$ is an upper bound, then we can represent the $\Phi_{\alpha}$ as $\left(X, \mathscr{B}_{\alpha}, \mu, \varphi\right)$, where the $\mathscr{B}_{\alpha}$ for $\alpha \in J$ form an increasing net of invariant sub- $\sigma$ algebras of $\mathscr{B}$. The mappings $\psi_{\alpha \alpha^{\prime}}$ and $\rho_{\alpha}$ become the identity mapping on $X$. Moreover, the inverse limit $\Phi$, if it exists, can be identified with $(X, \hat{\mathscr{B}}, \mu, \varphi)$, where $\bigcup_{\alpha \in J} \mathscr{B}_{\alpha} \subseteq \hat{\mathscr{B}} \subseteq \mathscr{B}$. We shall show now that this identification leads to a proof of the existence and uniqueness of the inverse limit for any bounded inverse system.

If $\mathscr{B}_{\alpha}(\alpha \in J)$ is any collection of sub- $\sigma$-algebras of a $\sigma$-algebra $\mathscr{B}$, we shall denote by $\bigvee_{\alpha \in J} \mathscr{B}_{\alpha}$ the smallest $\sigma$-algebra containing $\bigcup_{\alpha \in J} \mathscr{B}_{\alpha}$ and call it the join of the $\mathscr{B}_{\alpha}$. If $\Phi_{\alpha}(\alpha \in J)$ is a collection of factors of the dynamical system $\Phi$, say $\rho_{\alpha}: \Phi \rightarrow \Phi_{\alpha}$, this allows us to define the join $\hat{\Phi}=\bigvee_{\alpha \in J} \Phi_{\alpha}$ to be $\hat{\Phi}=\left(X, \bigvee_{\alpha \in J} \rho_{\alpha}^{-1}\left(\mathscr{B}_{\alpha}\right), \mu, \varphi\right)$. Note that the join $\bigvee_{\alpha \in J} \Phi_{\alpha}$ coincides with the product $\bigotimes_{\alpha \in J} \Phi_{\alpha}$ iff the collection $\left\{\rho_{\alpha}^{-1}\left(\mathscr{B}_{\alpha}\right): \alpha \in J\right\}$ is independent. We shall show that if $\left(J, \Phi_{\alpha}, \psi_{\alpha \alpha^{\prime}}\right)$ is an inverse system, then $\bigvee_{\alpha \in J} \Phi_{\alpha}=\operatorname{inv} \lim _{\alpha \in J} \Phi_{\alpha}$.

THEOREM 1. If $\left(J, \Phi_{\alpha}, \psi_{\alpha \alpha^{\prime}}\right)$ is an inverse system with upper bound $\Phi$, then inv $\lim _{\alpha \in J} \Phi_{\alpha}=\bigvee_{\alpha \in J} \Phi_{\alpha}$. In particular, the inverse limit, when it exists, is uniquely determined (up to isomorphism).

Proof. We may use the "internal characterization" of $\left(J, \Phi_{\alpha}, \psi_{\alpha \alpha^{\prime}}\right)$ whereby $\Phi=(X, \mathscr{B}, \mu, \varphi), \Phi_{\alpha}=\left(X, \mathscr{B}_{\alpha}, \mu, \varphi\right), \psi_{\alpha \alpha^{\prime}}=\rho_{\alpha}=$ identity. It is clear then that $\Phi=$ $\left(X, \bigvee_{\alpha \in J} \mathscr{B}_{\alpha}, \mu, \varphi\right)$ is an upper bound. We need only show that any other upper bound can be factored through $\Phi$. Let $\Phi^{\prime}=\left(X^{\prime}, \mathscr{B}^{\prime}, \mu^{\prime}, \varphi^{\prime}\right)$ be any other upper bound, relative say to the maps $\rho_{\alpha}^{\prime}: \Phi^{\prime} \rightarrow \Phi_{\alpha}$. Since each $\psi_{\alpha \alpha^{\prime}}$ is the identity on $X$, it follows that the maps $\rho^{\prime}=\rho_{\alpha}^{\prime}: X^{\prime} \rightarrow X$ are the same for each $\alpha \in J$. Moreover, $\rho^{\prime-1}\left(\bigcup_{\alpha \in J} \mathscr{B}_{\alpha}\right)=\bigcup_{\alpha \in J} \rho^{\prime-1}\left(\mathscr{B}_{\alpha}\right) \subseteq \mathscr{B}^{\prime}$. Thus $\rho^{\prime-1}\left(\bigvee_{\alpha \in J} \mathscr{B}_{\alpha}\right) \subseteq \mathscr{B}^{\prime}$ and $\Phi$ is a factor of $\Phi^{\prime}, \rho^{\prime}: \Phi^{\prime} \rightarrow \Phi$. Moreover, since each $\rho_{\alpha}$ is the identity on $X$, the proper diagrams commute, and we have shown that $\Phi$ is an inverse limit.

So far we have only shown that two inverse limits are weakly isomorphic. However, the above construction can be used now to yield an isomorphism. For suppose that $\Phi$ itself is an inverse limit. Then there must exist a map $\sigma: \Phi \rightarrow \Phi$ such that the diagram

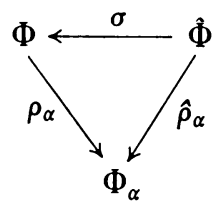

commutes, where $\rho_{\alpha}(x)=\hat{\rho}_{\alpha}(x)=x(x \in X)$. It follows that $\sigma(x)=x$. Measurability of $\sigma$ thus implies that $\mathscr{B} \subseteq \bigvee_{\alpha \in J} \mathscr{B}_{\alpha}$, and hence that they coincide. Returning to the external description of the inverse limit, we have shown that, if the upper bound $\Phi$ is an inverse limit, then $\bigcup_{\alpha \in J} \rho_{\alpha}^{-1}\left(\mathscr{B}_{\alpha}\right)$ is dense in $\mathscr{B}$. This, of course, implies that any two inverse limits are equivalent (isomorphic). 
Note that if it were not for our desire to pass back and forth from factors to invariant sub- $\sigma$-algebras, we could merely have insisted that all of our measure spaces be sufficiently decent to support "enough" measure-preserving point transformations. This is done, for instance, in [14] and elsewhere by restricting attention to Lebesgue spaces and defining factor transformations on the so-called measurable partitions. However, some important dynamical systems that we shall want to consider are not defined on Lebesgue spaces. (See, for example, Theorem 3 and the paper [3].) If such a policy were pursued, then the uniqueness theorem above would yield a point isomorphism. In this sense it is stronger than the uniqueness theorem of Choksi [4].

The question of existence of the inverse limit is somewhat more difficult. The usual approach is to define the inverse limit set

$$
X_{\infty}=\left\{x \in \underset{\alpha \in J}{X} X_{\alpha}: \psi_{\alpha \alpha^{\prime}} x_{\alpha^{\prime}}=x_{\alpha} \text { for all } \alpha, \alpha^{\prime} \in J, \alpha<\alpha^{\prime}\right\},
$$

define the projections $\rho_{\alpha}: X_{\infty} \rightarrow X_{\alpha}$ in the obvious way and attempt to extend the measures $\mu_{\alpha} \circ \rho_{\alpha}$ from $\bigcup_{\alpha \in J} \rho_{\alpha}^{-1}\left(\mathscr{B}_{\alpha}\right)$ to $\bigvee_{\alpha \in J} \rho_{\alpha}^{-1}\left(\mathscr{B}_{\alpha}\right)$. However, it is known (see e.g. [7, p. 214]) that this is not always possible even when each $\varphi_{\alpha}$ is the identity and the measurable spaces $\left(X_{\alpha}, \mathscr{B}_{\alpha}\right)$ are partial products. However, our "free substitution rule" makes it possible to assert that the inverse limit in our sense always exists. Note that Theorem 1 implies that the inverse limit of a sequence of Lebesgue systems is a Lebesgue system. In general the inverse limit of Lebesgue systems need not be a Lebesgue system.

THEOREM 2. The inverse limit of any inverse system of dynamical systems exists.

Proof. In [4] J. R. Choksi has shown (in the proof of Theorem 5.2) that the given system can be replaced by a system $\left(J, \Phi_{\alpha}^{1}, \psi_{\alpha \alpha^{\prime}}^{1}\right)$ with the measure algebras $\mathscr{B}_{\alpha}(\mu)$ and $\mathscr{B}_{\alpha}^{1}\left(\mu^{1}\right)$ isomorphic under a system of isomorphisms $\sigma_{\alpha}$ that commute with the maps $\psi_{\alpha \alpha^{\prime}}$ and $\psi_{\alpha \alpha^{\prime}}^{1}$, i.e. $\psi_{\alpha \alpha^{\prime}}^{1} \sigma_{\alpha^{\prime}}=\sigma_{\alpha} \psi_{\alpha \alpha^{\prime}}$, and such that (1) the spaces $X_{\alpha}^{1}$ are compact Hausdorff and (2) the maps $\psi_{\alpha \alpha^{\prime}}^{1}$ are continuous. His Theorem 2.2 then gives the existence of an inverse limit measure space $(X, \mathscr{B}, \mu)$, with $X$ being the inverse limit set of the system $\left(J, \Phi_{\alpha}^{1}, \psi_{\alpha \alpha^{\prime}}^{1}\right)$. Next we observe that $\varphi_{\alpha}^{-1}(A)$ $=\sigma_{\alpha} \varphi_{\alpha}^{-1} \sigma_{\alpha}^{-1}(A)$ defines a measure-preserving set mapping in $X_{\alpha}^{1}$. Since $X_{\alpha}^{1}$ is a "decent" measure space, $\varphi_{\alpha}^{-1}$ must be essentially the inverse of a point mapping $\varphi_{\alpha}^{1}: X_{\alpha}^{1} \rightarrow X_{\alpha}^{1}$. Moreover, the appropriate diagrams commute, and so $\psi_{\alpha \alpha^{\prime}}^{1}: \Phi_{\alpha^{\prime}}^{1} \rightarrow \Phi_{\alpha}^{1}$ whenever $\alpha<\alpha^{\prime}$. Finally, we define $\varphi: X \rightarrow X$ by $\varphi(x)=y$ where $y_{\alpha}=\varphi_{\alpha}^{1} x_{\alpha}$ for each $x \in X$. It follows that $\Phi=(X, \mathscr{B}, \mu, \varphi)$ is the inverse limit of the system $\left(J, \Phi_{\alpha}^{1}, \psi_{\alpha \alpha^{\prime}}^{1}\right)$ and hence of the system $\left(J, \Phi_{\alpha}, \psi_{\alpha \alpha^{\prime}}\right)$.

3. Examples. The most obvious example of an inverse limit is the direct product defined in the first section. In this case we can take $I$ to be the set of finite subsets of $J$, directed by inclusion. Then

$$
\bigotimes_{\alpha \in J} \Phi_{\alpha}=\operatorname{inv}_{\left(\alpha_{1}, \ldots, \alpha_{n}\right) \in I} \Phi_{\alpha_{1}} \otimes \Phi_{\alpha_{2}} \otimes \cdots \otimes \Phi_{\alpha_{n}}
$$


While this is a relatively uninteresting observation, it is useful in extending the addition formula for entropy (see §5). In addition, this example is, of course, the prototype for the notion of inverse limit.

A somewhat more interesting example is the following. For each $n=1,2,3, \ldots$ let $Y_{n}=\left\{0,1, \ldots, k_{n}-1\right\}$ be a finite cyclic group of order $k_{n}$. Let $\mathscr{A}_{n}$ denote all the subsets of $Y_{n}$, and let $v_{n}=\left\{p_{n 0}, p_{n 1}, \ldots, p_{n, k_{n}-1}\right\}$ be a probability measure. We define $X_{n}=Y_{1} \times \cdots \times Y_{n}, \mathscr{B}_{n}=\mathscr{A}_{1} \times \cdots \times \mathscr{A}_{n}, \mu_{n}=\nu_{1} \times \cdots \times v_{n}$ and

$$
\begin{aligned}
\varphi_{n}\left(x_{1}, \ldots, x_{n}\right) & =\left(x_{1}+1, x_{2}, \ldots, x_{n}\right) \text { if } x_{1}<k_{1}-1, \\
& =\left(0, \ldots, 0, x_{j}+1, x_{j+1}, \ldots, x_{n}\right) \text { if } j \text { is the smallest integer for } \\
& =(0,0, \ldots, 0) \text { if } x_{j}=k_{j}-1 \text { for all } j . \quad \text { which } x_{j}<k_{j}-1,
\end{aligned}
$$

Then $\varphi_{n}$ is an ergodic measure-preserving (invertible) transformation iff $p_{n j}=1 / k_{n}$ is independent of $j$. The sequence $\Phi_{n}=\left(X_{n}, \mathscr{B}_{n}, \mu_{n}, \varphi_{n}\right)$ is an inverse system with respect to the projection maps

$$
\psi_{n, n+m}\left(x_{1}, \ldots, x_{n+m}\right)=\left(x_{1}, \ldots, x_{n}\right) .
$$

The inverse limit of the sequence is $\Phi=(X, \mathscr{B}, \mu, \varphi)$, where $X=\times_{n=1}^{\infty} X_{n}, \mathscr{B}=$ $\times_{n=1}^{\infty} \mathscr{B}_{n}, \mu=\times_{n=1}^{\infty} \mu_{n}$, and $\varphi$ is defined like $\varphi_{n}$ with the obvious modifications.

It is not hard to see that the $X$ of this example, considered as the inverse limit of a sequence of topological groups, is a monothetic, compact abelian group with topological generator $a=(1,0,0, \ldots)$, and that $\varphi(x)=x+a$. Thus this example is a special case of the following theorem.

THEOREM 3. Any ergodic dynamical system with discrete spectrum (see [6]) is the inverse limit of direct products of ergodic translations of the unit interval (addition mod 1) and cyclic.permutations of finite sets.

Proof. The pertinent facts about duality of topological groups may be found, for example, in [12]. It is proved in [6] that such a system is isomorphic to translation by a topological generator $a$ on a monothetic, compact abelian group $G$. Let $\Gamma=\hat{G}$ be the dual of the group $G$. Then $\Gamma$ is discrete, and the mapping $\psi^{*}$, defined by $\psi^{*} \gamma=\gamma(a)$, is a one-to-one homomorphism of $\Gamma$ onto a subgroup of $K_{d}$, the circle group with the discrete topology. Thus we may assume that $\Gamma \subseteq K_{d}$ (in which case $\psi^{*}$ is the identity map). Suppose that $\Gamma_{1} \subseteq \Gamma$ is finitely generated. Then $\Gamma_{1}=\left(\alpha_{1}\right) \oplus\left(\alpha_{2}\right) \oplus \cdots \oplus\left(\alpha_{n}\right)$ is a direct sum (equals direct product) of cyclic groups with generators $\alpha_{j} \in K_{d}$. Thinking of $K_{d}$ now as the unit interval with addition $\bmod 1$, each $\alpha_{j}$ is either rational, hence of finite order, or irrational, and so a topological generator of $K$ (usual topology). (As a matter of fact, not more than one $\alpha_{j}$ can be rational.) In the first case, the dual of $\left(\alpha_{j}\right)$ is isomorphic to $\left(\alpha_{j}\right)$, that is to the finite cyclic group $Z_{t}$ of integers $\bmod t$ for some $t$, and in the second case, since $\left(\alpha_{j}\right)$ is infinite cyclic, to $K$. Thus the dual $G_{1}$ of $\Gamma_{1}$ is a direct product of such 
factors. Since $\Gamma_{1} \subseteq \Gamma$, it follows that $G_{1}$ is a homomorphic image (in the sense of topological groups) of $G$. Let $\rho_{1}: G \rightarrow G_{1}$ be the homomorphism. Then $\rho_{1}$ is a measure-preserving (Haar measure in each case) mapping of $G$ onto $G_{1}$. It induces the factor dynamical system $\Phi_{1}$, where $\varphi_{1}\left(\rho_{1} x\right)=\rho_{1}(\varphi x)$. Since $\varphi(x)=x+a$, this gives

$$
\varphi_{1}\left(\rho_{1} x\right)=\rho_{1}(x+a)=\rho_{1}(x)+\rho_{1}(a) .
$$

Thus $\varphi_{1}$ is translation on $G_{1}$ by $\rho_{1}(a)$.

Let us calculate $\rho_{1}(a)$. Since $\rho_{1}$ is the adjoint of the restriction of the embedding map $\rho_{1}^{*}: \Gamma_{1} \rightarrow \Gamma \subseteq K_{d}$, we have for each $\gamma \in \Gamma_{1}$ that

$$
\gamma\left(\rho_{1}(a)\right)=\rho_{1}^{*} \gamma(a)=\gamma(a)=\psi^{*} \gamma=\gamma .
$$

Thus $\rho_{1}(a)$, considered as an element of $\left(\alpha_{1}\right)^{\wedge} \otimes\left(\alpha_{2}\right)^{\wedge} \otimes \cdots \otimes\left(\alpha_{n}\right)^{\wedge}$ is $\alpha_{1} \otimes \alpha_{2}$ $\otimes \cdots \otimes \alpha_{n}$. It follows that $\varphi_{1}$ is a direct product of translations of the type described in the theorem.

Finally, since $\Gamma$ is the union (equals inductive limit) of its finitely generated subgroups, it follows that $G$ is the inverse limit of factors of the type $G_{1}$. From this it follows immediately that $\Phi$ is the inverse limit of the corresponding factors $\Phi_{1}$.

One more example of particular importance is due to V. A. Rohlin [10]. Let $\Phi=(X, \mathscr{B}, \mu, \varphi)$ be a dynamical system, where $\varphi$ is, in general, not invertible. In particular, if $\bigcap_{n=0}^{\infty} \varphi^{-n \mathscr{B}}$ is trivial, $\Phi$ is called exact. Rohlin defines the natural extension $\hat{\Phi}$ of $\Phi$ in a way that is equivalent to the following. For each $n=1,2, \ldots$ let $\Phi_{n}=\Phi$, and for each $k=1,2, \ldots$ let $\psi_{n, n+k}: \Phi_{n+k} \rightarrow \Phi_{n}$ be defined by $\psi_{n, n+k}$ $=\varphi^{k}$. The inverse limit $\hat{\Phi}$ of this sequence is an invertible dynamical system, called the natural extension of $\Phi$. Rohlin actually considered only Lebesgue systems $\Phi$, in which case $\Phi$ is also a Lebesgue system. He showed that an invertible dynamical system $\Phi$ has completely positive entropy (that is, every factor of $\Phi$ has a noninvertible factor) iff $\Phi$ is the natural extension of an exact system (see [11]).

To see that $\Phi$ is invertible, note that by the proof of Theorem 2 we can assume that $\hat{X}$ is the inverse limit set

But then

$$
\hat{X}=\left\{x \in \underset{n=1}{\infty} X_{n}: x_{n}=\varphi x_{n+1} \text { for each } n\right\} .
$$

$$
\hat{\varphi}\left(x_{1}, x_{2}, x_{3}, \ldots\right)=\left(\varphi x_{1}, \varphi x_{2}, \varphi x_{3}, \ldots\right)=\left(\varphi x_{1}, x_{1}, x_{2}, \ldots\right) .
$$

Thus $\hat{\varphi}$ is one-to-one, and its inverse

$$
\hat{\varphi}^{-1}\left(x_{1}, x_{2}, x_{3}, \ldots\right)=\left(x_{2}, x_{3}, x_{4}, \ldots\right)
$$

is also measurable. That is, $\hat{\Phi}$ is invertible. Of course, if $\Phi$ is invertible, then $\hat{\Phi}$ is isomorphic to $\Phi$.

4. Properties of the inverse limit. In this section, we make some elementary but useful observations about inverse limits. 
THEOREM 4. Let $\left(J, \Phi_{\alpha}, l_{\alpha \alpha^{\prime}}\right)$ be an inverse system of dynamical systems, and let $J_{0} \subseteq J$ have the property that for each $\alpha \in J$ there is $a \beta \in J_{0}$ such that $\alpha<\beta$. Then $\left(J_{0}, \Phi_{\alpha}, \psi_{\alpha \alpha^{\prime}}\right)$ is an inverse system, and inv $\lim _{\alpha \in J} \Phi_{\alpha}=\operatorname{inv} \lim _{\alpha \in J_{0}} \Phi_{\alpha}$.

Proof. Since $\bigvee_{\alpha \in J} \Phi_{\alpha}$ is clearly the same as $\bigvee_{\alpha \in J_{0}} \Phi_{\alpha}$, the result is immediate from the internal characterization of the $\Phi_{\alpha}$ as factors of inv $\lim _{\alpha \in J} \Phi_{\alpha}$. Externally, the theorem is simply the observation that we can fill in "gaps" in the system of projections by setting $\rho_{\alpha}=\psi_{\alpha \beta} \rho_{\beta}$.

THEOREM 5. If $\Sigma_{\alpha} \mid \Phi_{\alpha}$ for each $\alpha \in J$, then inv $\lim _{\alpha \in J} \sum_{\alpha} \mid \operatorname{inv} \lim _{\alpha \in J} \Phi_{\alpha}$. In particular, if $\varphi_{\alpha}$ and $\psi_{\alpha}$ are commuting transformations on $X_{\alpha}$ for each $\alpha \in J$, then

also commute.

$$
\varphi=\underset{\alpha \in J}{\operatorname{inv}} \lim \varphi_{\alpha} \quad \text { and } \quad \psi=\underset{\alpha \in J}{\operatorname{inv} \lim } \psi_{\alpha}
$$

THEOREM 6. If $\Phi_{\alpha}^{1}$ and $\Phi_{\alpha}^{2}$ are factors of $\Phi_{\alpha}$ for each $\alpha \in J$, then

$$
\operatorname{inv} \lim _{\alpha \in J}\left(\Phi_{\alpha}^{1} \vee \Phi_{\alpha}^{2}\right)=\left(\underset{\alpha \in J}{\operatorname{inv}} \lim \Phi_{\alpha}^{1}\right) \vee\left(\underset{\alpha \in J}{\operatorname{inv} \lim } \Phi_{\alpha}^{2}\right),
$$

where the latter join is as factors of inv $\lim _{\alpha \in J} \Phi_{\alpha}$. In particular, inv $\lim _{\alpha \in J}\left(\Phi_{\alpha}^{1} \otimes \Phi_{\alpha}^{2}\right)$ $=\left(\right.$ inv $\left.\lim _{\alpha \in J} \Phi_{\alpha}^{1}\right) \otimes\left(\operatorname{inv} \lim _{\alpha \in J} \Phi_{\alpha}^{2}\right)$. Moreover, if $\Phi_{\alpha}^{1}$ and $\Phi_{\alpha}^{2}$ are disjoint, in the sense of Furstenberg [5], for each $\alpha \in J$, then inv $\lim _{\alpha \in J} \Phi_{\alpha}^{1}$ and inv $\lim _{\alpha \in J} \Phi_{\alpha}^{2}$ are disjoint.

The proofs of Theorems 5 and 6 are routine verifications and will be omitted.

Theorem 7. The inverse limit inv $\lim _{\alpha \in J} \Phi_{\alpha}$ is (1) ergodic, (2) weakly mixing, (3) mixing (of any order) iff each $\Phi_{\alpha}$ has the same property.

Proof. As in the proof of Theorem 1, we may assume that $\Phi=\operatorname{inv} \lim _{\alpha \in J} \Phi_{\alpha}$ $=(X, \mathscr{B}, \mu, \varphi), \Phi_{\alpha}=\left(X, \mathscr{B}_{\alpha}, \mu, \varphi\right)$, where the $\mathscr{B}_{\alpha}(\alpha \in J)$ form an increasing net, and $\mathscr{B}=\bigvee_{\alpha \in J} \mathscr{B}_{\alpha}$. Now it is well known that $\Phi$ is ergodic iff

$$
\lim _{n \rightarrow \infty} \frac{1}{n} \sum_{k=0}^{n-1} \mu\left(A \cap \varphi^{-k} B\right)=\mu(A) \mu(B)
$$

for each pair $A, B \in \mathscr{B}$. As a matter of fact, it is sufficient to show that (1) holds for each pair $A, B \in \mathscr{B}_{0}$, where $\mathscr{B}_{0}$ is some algebra dense in $\mathscr{B}$. For if $\mu\left(A \Delta A_{0}\right)<\varepsilon$ and $\mu\left(B \Delta B_{0}\right)<\varepsilon$, then

$$
\begin{aligned}
\left|\mu\left(A \cap \varphi^{-k} B\right)-\mu\left(A_{0} \cap \varphi^{-k} B_{0}\right)\right| & \leqq \mu\left[\left(A \cap \varphi^{-k} B\right) \Delta\left(A_{0} \cap \varphi^{-k} B_{0}\right)\right] \\
& \leqq \mu\left[\left(A \Delta A_{0}\right) \cup\left(\varphi^{-k} B \Delta \varphi^{-k} B_{0}\right)\right]<2 \varepsilon
\end{aligned}
$$

for each $k$. Taking $\mathscr{B}_{0}=\bigcup_{\alpha \in J} \mathscr{B}_{\alpha}$ shows that $\Phi$ is ergodic iff each $\Phi_{\alpha}$ is ergodic. (An interesting alternate proof uses the definition of ergodicity and the martingale theorem. The above proof, of course, uses the ergodic theorem.) A similar argument shows that $\Phi$ is weakly mixing or mixing iff each $\Phi_{\alpha}$ is. Alternatively, $\Phi$ is weakly mixing iff $\Phi \otimes \Phi$ is ergodic, which by Theorem 6 and the first part of this theorem is true iff each $\Phi_{\alpha} \otimes \Phi_{\alpha}$ is ergodic, that is iff each $\Phi_{\alpha}$ is weakly mixing. 
5. Entropy. In this section we discuss the entropy of an abstract dynamical system $\Phi$, particularly as it relates to inverse limits. In many cases, the calculation of the entropy of $\Phi$ is accomplished by representing $\Phi=\operatorname{inv}_{\alpha \in J} \Phi_{\alpha}$ as an inverse limit, calculating the entropy of $\Phi_{\alpha}$ for each $\alpha \in J$, and passing to the limit. This is the case, for example, in [10], where the entropy of the natural extension of $\Phi$ is shown to be equal to the entropy of $\Phi$, in [1] and in [13], where the entropy of systems with quasi-discrete spectrum and quasi-periodic spectrum, respectively, is shown to be zero. In each of these cases, the inverse systems involved are sequences. However, Theorem 3 of $\S 3$ and the results in [3] show that the same approach can be fruitful for more general inverse systems.

The definition and basic properties of the entropy of a dynamical system may be found, for example, in [2], where the fundamental lemma below is also proved.

Let $\Phi=(X, \mathscr{B}, \mu, \varphi)$ be a dynamical system. For each finite algebra $\mathscr{A} \subseteq \mathscr{B}$, let $\mathscr{A}$ denote the collection of its atoms. Thus $\dot{\mathscr{A}}=\left\{A_{1}, \ldots, A_{n}\right\}$ is a partition of $X$, in the sense that its elements are pairwise disjoint and $\mu\left(X \sim \bigcup_{j=1}^{n} A_{j}\right)=0$. For such an algebra $\mathscr{A}$ let

$$
H(\mathscr{A})=-\sum_{A \in \mathscr{A}} \mu(A) \log \mu(A)
$$

and

$$
h(\varphi, \mathscr{A})=\lim _{n \rightarrow \infty} \frac{1}{n} H\left(\mathscr{A} \vee \varphi^{-1} \mathscr{A} \vee \cdots \vee \varphi^{-(n-1)} \mathscr{A}\right)
$$

We define the entropy of the dynamical system $\Phi$ to be the number

$$
h(\Phi)=\sup _{\mathscr{A} \subseteq \mathscr{D}} h(\varphi, \mathscr{A}) .
$$

It then follows (see [2]) that $0 \leqq h(\Phi) \leqq \infty, h\left(\Phi^{n}\right)=h(\Phi)|n|$ (for positive $n$ in general, for positive or negative $n$ in case $\Phi$ is invertible), $h\left(\Phi_{1} \otimes \Phi_{2}\right)=h\left(\Phi_{1}\right)+h\left(\Phi_{2}\right)$, and that $h\left(\Phi_{1}\right) \leqq h\left(\Phi_{2}\right)$ whenever $\Phi_{1} \mid \Phi_{2}$.

Aside from these "arithmetical" properties of $h$, the principal tool in calculating the entropy of familiar dynamical systems has been the following theorem of Sinaĭ and various extensions of it (see [2, Theorem 7.3 and its corollaries]).

THEOREM (SINAI). If $\mathscr{A}$ is a finite subalgebra of $\mathscr{B}$ such that $(1) \bigvee_{n=0}^{\infty} \varphi^{-n} \mathscr{A}=\mathscr{B}$ or (2) $\Phi$ is invertible and $\bigvee_{n=-\infty}^{\infty} \varphi^{-n} \mathscr{A}=\mathscr{B}$, then $h(\Phi)=h(\varphi, \mathscr{A})$.

Note that, for any finite algebra $\mathscr{A} \subseteq \mathscr{B}$, the $\sigma$-algebra $\mathscr{B}_{1}=\bigvee_{n=0}^{\infty} \varphi^{-n} \mathscr{A}$ is invariant, and $h(\varphi, \mathscr{A})=h\left(\Phi_{1}\right)$, where $\Phi_{1}=\left(X, \mathscr{B}_{1}, \mu, \varphi\right)$ is a factor of $\Phi$. Thus the various extensions of Sinai's theorem become special cases of our Theorem 8 below. We shall make use of the following lemma [2, p. 89], whose proof can be made independent of the Sinair theorem.

LeMma. Let $\mathscr{B}_{0}$ be a dense subalgebra of $\mathscr{B}$. Then

$$
h(\Phi)=\sup _{\mathscr{A} \subseteq \mathscr{\mathscr { O }}_{0}} h(\varphi, \mathscr{A}) .
$$


THEOREM 8. If $\Phi=\operatorname{inv} 1: \mathrm{n}_{\alpha \in J} \Phi_{\alpha}$, then

$$
h(\Phi)=\lim _{\alpha} h\left(\Phi_{\alpha}\right)
$$

REMARK. Of course, by the monotone character of the function $h$ with regard to factors, the numbers $h\left(\Phi_{\alpha}\right)(\alpha \in J)$ form an increasing net, and so $\lim _{\alpha} h\left(\Phi_{\alpha}\right)$ $=\sup _{\alpha \in J} h\left(\Phi_{\alpha}\right) \leqq+\infty$ exists.

Proof. We may assume without loss of generality that $\Phi=(X, \mathscr{B}, \mu, \varphi)$ and $\Phi_{\alpha}=\left(X, \mathscr{B}_{\alpha}, \mu, \varphi\right)$ for each $\alpha \in J$. Since $\Phi_{\alpha} \mid \Phi$, we have $h\left(\Phi_{\alpha}\right) \leqq h(\Phi)$ for each $\alpha$, and so $h(\Phi) \geqq \sup _{\alpha \in J} h\left(\Phi_{\alpha}\right)=\lim _{\alpha} h\left(\Phi_{\alpha}\right)$. To prove the reverse inequality, we use the lemma with $\mathscr{B}_{0}=\bigcup_{\alpha \in J} \mathscr{B}_{\alpha}$. Then if $\mathscr{A}_{0}$ is a finite subalgebra of $\mathscr{B}_{0}$, there must exist an $\alpha \in J$ with $\mathscr{A}_{0} \subseteq \mathscr{B}_{\alpha}$. But then

$$
h\left(\varphi, \mathscr{A}_{0}\right) \leqq \sup _{\mathscr{A} \subseteq \mathscr{B}_{\alpha}} h(\varphi, \mathscr{A})=h\left(\Phi_{\alpha}\right) .
$$

It follows that

$$
h(\Phi)=\sup _{\mathscr{A} \subseteq \mathscr{B}_{0}} h(\varphi, \mathscr{A}) \leqq \sup _{\alpha \in J} h\left(\Phi_{\alpha}\right),
$$

and the proof is complete.

CoRollary 1. If $\Phi=\bigotimes_{\alpha \in J} \Phi_{\alpha}$, then

$$
h(\Phi)=\sum_{\alpha \in J} h\left(\Phi_{\alpha}\right)
$$

This sum is, of course, interpreted to be $+\infty$ if more than a countable number of the $\Phi_{\alpha}$ have positive entropy (or if the sum diverges).

From Theorem 3 we obtain the following corollary, which of course is well known.

COROllary 2. Any ergodic dynamical system with discrete spectrum has zero entropy.

As indicated earlier, similar analyses of systems with quasi-discrete spectrum or quasi-periodic spectrum reveal that they also have zero entropy. We might note also the following theorem of Rohlin [10].

COROLlary 3. If $\Phi$ is the natural extension of $\Phi$, then $h(\Phi)=h(\Phi)$.

REMARK. An immediate consequence of Theorem 6 is that any inverse limit of dynamical systems with zero entropy has zero entropy. At the opposite extreme, it is proved in [11] that the inverse limit of a sequence of Lebesgue systems with completely positive entropy (every nontrivial factor has positive entropy) has completely positive entropy. It seems likely that this is true for arbitrary inverse limits of systems with completely positive entropy. However, the present author has been unable to demonstrate this. 
6. Weak isomorphism. Following Sinaĭ [14] we have defined two dynamical systems $\Phi_{1}$ and $\Phi_{2}$ to be weakly isomorphic if each is a factor of the other. While it is not known whether this implies isomorphism of $\Phi_{1}$ and $\Phi_{2}$, we shall now show that whenever $\Phi_{1}$ and $\Phi_{2}$ are weakly isomorphic, there exist dynamical systems $\hat{\Phi}_{1}$ and $\hat{\Phi}_{2}$, such that $\Phi_{i}$ is a factor of $\hat{\Phi}_{i}(i=1,2)$ with the same entropy, and a "lifting" of the weak isomorphism to an isomorphism of $\hat{\Phi}_{1}$ and $\hat{\Phi}_{2}$.

Theorem 9. Suppose that $\sigma_{1}: \Phi_{1} \rightarrow \Phi_{2}$ and $\sigma_{2}: \Phi_{2} \rightarrow \Phi_{1}$. Define $\psi_{1}=\sigma_{2} \sigma_{1}: \Phi_{1} \rightarrow \Phi_{1}$ and $\psi_{2}=\sigma_{1} \sigma_{2}: \Phi_{2} \rightarrow \Phi_{2}$. Let $J$ be the positive integers, and for each $n \in J$, let $\Phi_{n}^{1}=\Phi_{1}$, $\Phi_{n}^{2}=\Phi_{2}, \psi_{n, n+k}^{1}=\psi_{1}^{k}, \psi_{n, n+k}^{2}=\psi_{2}^{k}$. Then $\hat{\Phi}_{1}=\operatorname{inv} \lim _{n \in J}\left(\Phi_{n}^{1}, \psi_{n n^{\prime}}^{1}\right)$ is isomorphic to $\hat{\Phi}_{2}=\operatorname{inv} \lim _{n \in J}\left(\Phi_{n}^{2}, \psi_{n n^{\prime}}^{2}\right)$. In fact, there exist isomorphisms $\hat{\sigma}_{1}: \Phi_{1} \rightarrow \hat{\Phi}_{2}$ and $\hat{\sigma}_{2}: \hat{\Phi}_{2} \rightarrow \hat{\Phi}_{1}$ such that $\hat{\sigma}_{2} \hat{\sigma}_{1}$ is the natural extension of $\psi_{1}$.

Proof. The proof may best be summarized in the following diagram.

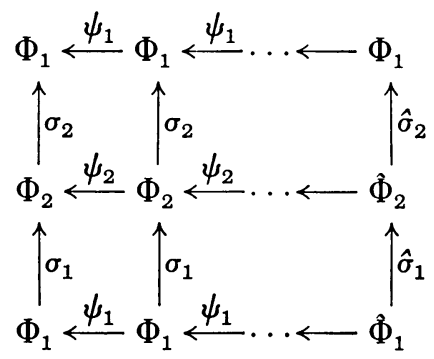

The existence of the isomorphism follows from Theorems 1 and 4 and the observation that the first and second rows of the above diagram represent subsequences of the system

$$
\Phi_{1} \stackrel{\sigma_{2}}{\longleftarrow} \Phi_{2} \stackrel{\sigma_{1}}{\longleftarrow} \Phi_{1} \stackrel{\sigma_{2}}{\longleftarrow} \Phi_{2} \stackrel{\sigma_{1}}{\longleftarrow} \ldots \longleftarrow \Phi
$$

so that $\Phi, \hat{\Phi}_{1}$ and $\hat{\Phi}_{2}$ are all isomorphic. The mappings $\hat{\sigma}_{1}$ and $\hat{\sigma}_{2}$ in the first diagram above are defined in the obvious way (as in the second half of Theorem 5), and the fact that $\hat{\sigma}_{2} \hat{\sigma}_{1}$ is the natural extension $\hat{\psi}_{1}$ of $\psi_{1}$ is obtained by deleting the middle row of this diagram. Since $\hat{\psi}_{1}$ is invertible, so are $\hat{\sigma}_{1}$ and $\hat{\sigma}_{2}$.

\section{BIBLIOGRAPHY}

1. L. M. Abramov, Metric automorphisms with quasi-discrete spectrum, Izv. Akad. Nauk SSSR Ser. Mat. 26 (1962), 513-530; English transl., Amer. Math. Soc. Transl. (2) 39 (1964), 37-56. MR 26 \#606.

2. P. Billingsley, Ergodic theory and information, Wiley, New York, 1965. MR 33 \#254.

3. J. R. Brown, A universal model for dynamical systems with quasi-discrete spectrum, Bull. Amer. Math. Soc. 75 (1969), 1028-1030. MR 39 \#5770.

4. J. R. Choksi, Inverse limits of measure spaces, Proc. London Math. Soc. (3) 8 (1958), 321-342. MR 20 \#3251.

5. H. Furstenberg, Disjointness in ergodic theory, minimal sets, and a problem in Diophantine approximation, Math. Systems Theory 1 (1967), 1-49. MR 35 \#4369. 
6. P. R. Halmos, Lectures on ergodic theory, Publ. Math. Soc. Japan, no. 3, Math. Soc. Japan, Tokyo, 1956. MR 20 \#3958.

7. —_, Measure theory, Van Nostrand, Princeton, N. J., 1950. MR 11, 504.

8. P. R. Halmos and J. von Neumann, Operator methods in classical mechanics. II, Ann. of Math. (2) 43 (1942), 332-350. MR 4, 14.

9. D. Maharam, On homogeneous measure algebras, Proc. Nat. Acad. Sci. U.S.A. 28 (1942), 108-111. MR 4, 12.

10. V. A. Rohlin, Exact endomorphisms of a Lebesgue space, Izv. Akad. Nauk SSSR Ser. Mat. 25 (1961), 499-530; English transl., Amer. Math. Soc. Transl. (2) 39 (1964), 1-36. MR 26 \#1423.

11. - Lectures on the entropy theory of transformations with invariant measure, Uspehi Mat. Nauk 22 (1967), no. 5 (137), 3-56= Russian Math. Surveys 22 (1967), no. 5, 1-52. MR 36 \#349.

12. W. Rudin, Fourier analysis on groups, Interscience Tracts in Pure and Appl. Math., no. 12, Interscience, New York, 1962. MR 27 \#2808.

13. T. L. Seethoff, Zero-entropy automorphisms of a compact abelian group, Oregon State University, Dept. of Math. Technical Report \#40, 1968.

14. J. G. Sinaĭ, On a weak isomorphism of transformations with invariant measure, Mat. Sb. 63 (105) (1964), 23-42; English transl., Amer. Math. Soc. Transl. (2) 57 (1966), 123-143. MR 28 \#5164b; MR 28, 1247.

Department of Mathematics, Oregon State University, Corvallis, Oregon 97331 